\title{
Création d'un site internet Européen de formation au sevrage tabagique
}

\author{
S. Catros (Bordeaux), M. Fenelon (Bordeaux), A. Rui (Birmingham), K. Ross (Birmingham), \\ D. Marcio (Saint-Jacques-De-Compostelle), B. Angel (Saint-Jacques-De-Compostelle), \\ M.D.S. Luis (Porto), S.M. Luis (Porto), P. Alberto (Milan), L. Giovanni (Milan), J.C. Fricain \\ (Bordeaux)
}

Introduction : Actuellement 208 millions de personnes consomment du tabac en Europe dont 12 millions en France. 650000 décès sont attribuables au tabac en Europe et environ 60000 décès par an sont imputables à cette consommation en France, ce qui en fait la première cause de décès évitable. Tous les acteurs de santé devraient être mobilisés pour lutter contre ce fléau. Le chirurgien dentiste doit se sentir concerné car il s'agit d'un enjeu général de santé publique mais aussi spécifique de santé bucco-dentaire. En effet le tabac est l'étiologie principale de certaines maladies graves de la muqueuse buccale : carcinomes épidermoïdes, leucoplasies, carcinome verruqueux (1). Le tabac est aussi un cofacteur favorisant les maladies parodontales (2). Enfin la consommation de tabac perturbe la cicatrisation après les actes de Chirurgie Orale et c'est une contre indication relative aux interventions chirurgie implantaire. Malgré ce constat, les chirurgiens dentistes restent peu impliqués dans l'accompagnement du sevrage tabagique (3). Les raisons sont probablement liées à un manque de formation et de connaissances qui limitent la mise en œuvre du sevrage tabagique auprès de leurs patients. Pour pallier ce manque, un projet européen a été mis en place grâce à un financement du programme ERASMUS + (4). L'objectif de ce projet est de former les professionnels de santé européens au sevrage tabagique grâce à plusieurs outils. Matériels et Méthodes : Le principal outil de formation sera un site internet diffusé en langue Anglaise, Française, Italienne, Portugaise, et Espagnole. Ce site permettra de fournir un outil d'e-learning afin de promouvoir l'implication des professionnels de santé et notamment des chirurgiens dentistes dans le sevrage tabagique. Par ailleurs, un livre téléchargeable reprenant le contenu du site internet sera également diffusé. Enfin, plusieurs actions de diffusion de l'information seront menées tout au long du projet au travers de communications lors de congrès scientifiques et d'articles dans des revues professionnelles. Résultats : Le site et le livre électronique sont accessibles gratuitement à partir du lien : http://smokingcessationtraining.com/ en/home/ en langue anglaise. La version française sera publiée en ligne dans le premier semestre 2018. Le site a été réalisé sous la coordination de Rui Albuquerque (Birmingham Dental Hospital UK) avec la collaboration de Ross Keat (Birmingham Dental Hospital UK), Jean-Christophe Fricain et Sylvain Catros (Université de Bordeaux, France), Marcio Diniz Freitas (Universidade de Santiago de Compostela Espagne), Luis Monteiro (Universitário de Ciências da Saúde Portugal), Giovanni Lodi (Universita di Milano, Italy). Conclusions : Ce projet devrait permettre d'augmenter le niveau de compétence des utilisateurs et un certificat sera délivré à ceux qui complèteront l'évaluation en ligne. Ce projet devrait permettre d'avoir un impact éducatif en formant les chirurgiens dentistes, un impact sur la santé des patients et un impact économique en réduisant les couts sociétaux induits par le tabagisme. 